\title{
R10 Technological innovation: adaptation of a latex agglutination test for anti-Rotavirus-A IgY employment
}

Natália Maria Lanzarini ${ }^{1}$, Gentil Arthur Lins Bentes Mendonça de Vasconcelos $^{1}$, Juliana Rodrigues Guimarães ${ }^{1}$, Alexandre dos Santos da Silva1, Marcos Bryan Heinemann², José Paulo Leite', Eduardo de Melo Volotão ${ }^{1}$, Luiz Guilherme Dias Heneine ${ }^{3}$, Marcelo Alves Pinto ${ }^{1}$

1 IOC, Fiocruz, Rio de Janeiro, RJ

2 UFMG

${ }^{3}$ FUNED

Introduction: The infection by rotavirus (RV) is responsible for approximately 453,000 deaths annually and approximately $40 \%$ of hospitalizations by diarrhea in children under five years worldwide, being the major cause of acute gastroenteritis in this population group. The development of a rapid method, inexpensive, sensitive and specific for rotavirus diagnosis is important from the disease because it allows the identification of outbreaks of the disease in the site of occurrence. The use of Immunoglobulin Y (IgY) antibody purified from egg yolk, has been grown in recent years, due to the advantageous features compared to immunoglobulin $\mathrm{G}$ ( $\mathrm{lgG}$ ), as a noninvasive recovery of antibodies and production in high concentrations.

Objective: The aim of this method was to adapt a diagnostic test by replacing the IgG capture antibody by specific IgY for RV-A antigen (LATEXY-ROTA).

Metodologia: For that, 09 laying hens were immunized with RV-A, the eggs were collected and IgY purified from egg yolk by polyethylene glycol 6,000, followed by purification by ion Exchange chromatography. The purified anti-IgY RV-A was covalently bound to polystyrene particles, being tested in a panel of positive and negative fecal samples previously determined by the Rotavirus Regional Reference Center of Comparative and Environmental Virology Laboratory (LVCA/IOCFIOCRUZ).

Results: A sensitivity of $75 \%$ and specificity of $85,7 \%$ was observed when the adapted test was compared to a commercial available enzyme immunoassay (golden standard). When compared to two commercial latex agglutination tests using the $\lg$ tested on the panel of samples, the LATEXY-ROTA had a sensitivity of $100 \%$ and specificity of $88.2 \%$.

Conclusion: Based on the obtained data, we suggest the feasibility of replacing the $\lg G$ by $\lg Y$ in the latex agglutination assay.

Keywords: Imunoglobulina Y, Rotavírus, Látex 